\title{
Crystal structure of (oxalato- $\left.\kappa^{2} O: O^{\prime}\right)-\left[\mu-1,1^{\prime}-(1,6\right.$-hexanediyl)bis- (imidazole)- $\kappa^{2} N$ : $\left.N^{\prime}\right]$ cobalt(II) hemihydrate, $\mathrm{Co}\left(\mathrm{C}_{2} \mathrm{O}_{4}\right)\left(\mathrm{C}_{12} \mathrm{H}_{18} \mathrm{~N}_{4}\right) \cdot 0.5 \mathrm{H}_{2} \mathrm{O}$
}

\author{
Lai-Ping Zhang ${ }^{\mathrm{I}}$, Dao-Cheng Xia ${ }^{\mathrm{II}}$ and Jian-Fang $\mathrm{Ma}^{* . I}$ \\ 1 Northeast Normal University, Department of Chemistry, Changchun 130024, P. R. China \\ II Yuncheng University, Department of Chemistry, Yuncheng 044000, P. R. China
}

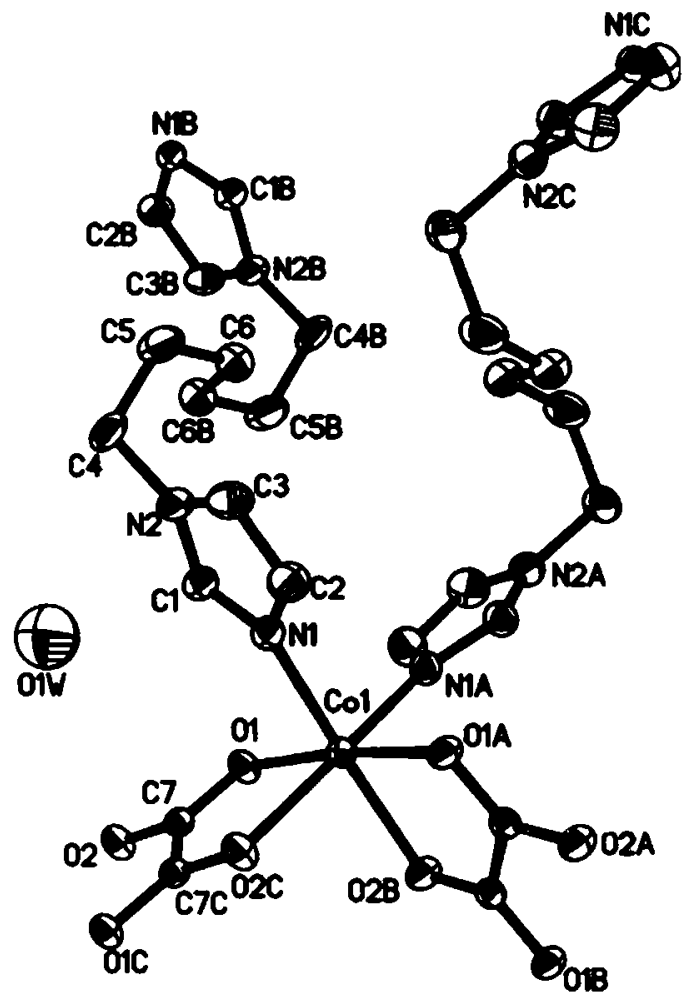

\section{Abstract}

$\mathrm{C}_{14} \mathrm{H}_{19} \mathrm{CoN}_{4} \mathrm{O}_{4.50}$, monoclinic, $\mathrm{C12} / \mathrm{c1}$ (no. 15), $a=12.653(6) \AA, b=13.888(6) \AA, c=9.403(6) \AA$, $\beta=100.49(3)^{\circ}, V=1624.7 \AA^{3}, Z=4, R_{g t}(F)=0.036$, $w R_{\text {ref }}\left(F^{2}\right)=0.086, T=293 \mathrm{~K}$.

\section{Source of material}

A mixture of $\mathrm{CoCl}_{2} \cdot 6 \mathrm{H}_{2} \mathrm{O}(0.238 \mathrm{~g}, 1 \mathrm{mmol})$ and $\mathrm{NaOH}(0.08 \mathrm{~g}$, $2 \mathrm{mmol}$ ) in water was stirred for $10 \mathrm{~min}$ at room temperature, and then 1,1'-(1,6-hexanediyl)bis(imidazole) (L, $0.432 \mathrm{~g}, 2 \mathrm{mmol})$ was added to the mixture. After stirring $0.5 \mathrm{~h}$, the precipitate was dissolved by dropwise addition of an aqueous solution of $\mathrm{NH}_{3}$ (14 M, $5 \mathrm{ml}$ ). Pink crystals were obtained after allowing the solution to stand at room temperature for several days.

\section{Experimental details}

All $\mathrm{H}$ atoms on $\mathrm{C}$ atoms were generated geometrically and refined as riding atoms with $d(\mathrm{C}-\mathrm{H})=0.93 \AA$ and $U_{\text {iso }}(\mathrm{H})=1.2 U_{\text {eq }}(\mathrm{C})$. The water $\mathrm{H}$ atoms were not included in the model.

\begin{abstract}
Discussion
In recent years, the bis(imidazole) ligands, as an important family of flexible $\mathrm{N}$-donor ligands, have attracted a great interest [1]. A series of fascinating carboxylate polymers based on $1,1^{\prime}-(1,4$ butanediyl)bis(imidazole) ligand have been reported [2]. By contrast, the exploratrion of carboxylate polymers constructed from $1,1^{\prime}-(1,6$-hexanediyl)bis(imidazole) $(\mathrm{L})$ is relatively less developed [3].

In the title crystal structure, each cobalt cation is six-coordinated by four $\mathrm{O}$ atoms from two oxalate anions and two $\mathrm{N}$ atoms from two $\mathrm{L}$ ligands forming a slightly distorted octahedron. The bond lengths of $\mathrm{Co}-\mathrm{O}$ and $\mathrm{Co}-\mathrm{N}$ are similar to the reported values [4]. The cobalt cations are connected by oxalate anions to form chains, which are further bridged by $L$ ligands to give a twodimensional layer.
\end{abstract}

Table 1. Data collection and handling.

\begin{tabular}{ll}
\hline Crystal: & pink block, size $0.12 \times 0.16 \times 0.23 \mathrm{~mm}$ \\
Wavelength: & Mo $K_{\alpha}$ radiation $(0.71069 \AA)$ \\
$\mu:$ & $10.85 \mathrm{~cm}^{-1}$ \\
Diffractometer, scan mode: & Bruker APEX CCD, $\omega$ \\
$2 \theta_{\max }:$ & $54.96^{\circ}$ \\
$N(h k l)_{\text {measured, }} N(h k l)_{\text {hnique }}:$ & 7856,1862 \\
Criterion for $I_{\text {obs }}, N(h k l)_{\mathrm{gt}}:$ & $I_{\mathrm{obs}}>2 \sigma\left(I_{\mathrm{obs}}\right), 1620$ \\
$N(\text { param })_{\text {refined: }}$ & 107 \\
Programs: & SHELXS-97 [5], SHELXL-97 [6]
\end{tabular}

Table 2. Atomic coordinates and displacement parameters (in $\AA^{2}$ ).

\begin{tabular}{lllllll}
\hline Atom & Site & Occ. & $x$ & $y$ & $z$ & $U_{\text {iso }}$ \\
\hline H(1) & $8 f$ & & 0.4531 & 0.2500 & 1.0001 & 0.049 \\
H(2) & $8 f$ & 0.2798 & 0.2789 & 0.6149 & 0.062 \\
H(3) & $8 f$ & 0.2025 & 0.1465 & 0.7336 & 0.068 \\
H(4A) & $8 f$ & 0.2394 & 0.0988 & 1.0315 & 0.071 \\
H(4B) & $8 f$ & 0.3594 & 0.1160 & 1.1056 & 0.071 \\
H(5A) & $8 f$ & 0.2916 & -0.0164 & 0.8806 & 0.074 \\
H(5B) & $8 f$ & & 0.3338 & -0.0449 & 1.0420 & 0.074 \\
H(6A) & $8 f$ & & 0.4636 & -0.0710 & 0.8964 & 0.068 \\
H(6B) & $8 f$ & 0.4639 & 0.0392 & 0.8621 & 0.068 \\
O(1W) & $4 e$ & 0.50 & $1 / 2$ & $0.2815(8)$ & $1 / 2$ & $0.153(4)$ \\
& & & & & & \\
\hline
\end{tabular}


Table 3. Atomic coordinates and displacement parameters (in $\AA^{2}$ ).

\begin{tabular}{|c|c|c|c|c|c|c|c|c|c|c|}
\hline Atom & Site & $x$ & $y$ & $z$ & $U_{11}$ & $U_{22}$ & $U_{33}$ & $U_{12}$ & $U_{13}$ & $U_{23}$ \\
\hline $\operatorname{Co}(1)$ & $4 e$ & $1 / 2$ & $0.39769(3)$ & $3 / 4$ & $0.0323(2)$ & $0.0277(2)$ & $0.0253(2)$ & 0 & $0.0057(1)$ & 0 \\
\hline$C(1)$ & $8 f$ & $0.4005(2)$ & $0.2411(2)$ & $0.9182(2)$ & $0.041(1)$ & $0.040(1)$ & $0.042(1)$ & $-0.0061(9)$ & $0.011(1)$ & $0.0013(9)$ \\
\hline$C(2)$ & $8 f$ & $0.3055(2)$ & $0.2566(2)$ & $0.7080(3)$ & $0.057(2)$ & $0.054(1)$ & $0.042(1)$ & $-0.015(1)$ & $0.001(1)$ & $-0.005(1)$ \\
\hline$C(3)$ & $8 f$ & $0.2622(2)$ & $0.1832(2)$ & $0.7729(3)$ & $0.052(2)$ & $0.052(1)$ & $0.064(2)$ & $-0.020(1)$ & $0.008(1)$ & $-0.008(1)$ \\
\hline C(4) & $8 f$ & $0.3129(2)$ & $0.0998(2)$ & $1.0150(3)$ & $0.061(2)$ & $0.050(1)$ & $0.079(2)$ & $-0.003(1)$ & $0.044(2)$ & $0.014(1)$ \\
\hline$C(5)$ & $8 f$ & $0.3421(2)$ & $0.0012(2)$ & $0.9672(4)$ & $0.060(2)$ & $0.046(1)$ & $0.084(2)$ & $-0.005(1)$ & $0.024(2)$ & $0.012(1)$ \\
\hline$C(6)$ & $8 f$ & $0.4555(2)$ & $-0.0075(2)$ & $0.9360(3)$ & $0.068(2)$ & $0.048(1)$ & $0.059(2)$ & $0.003(1)$ & $0.023(1)$ & $-0.002(1)$ \\
\hline$C(7)$ & $8 f$ & $0.5485(2)$ & $0.4696(1)$ & $1.0386(2)$ & $0.0274(9)$ & $0.0282(9)$ & $0.0259(9)$ & $-0.0009(7)$ & $0.0033(7)$ & $0.0019(7)$ \\
\hline $\mathrm{N}(1)$ & $8 f$ & $0.3924(1)$ & $0.2930(1)$ & $0.7993(2)$ & $0.040(1)$ & $0.0372(9)$ & $0.0346(9)$ & $-0.0062(7)$ & $0.0101(8)$ & $-0.0018(7)$ \\
\hline $\mathrm{N}(2)$ & $8 f$ & $0.3233(2)$ & $0.1738(1)$ & $0.9065(2)$ & $0.046(1)$ & $0.038(1)$ & $0.053(1)$ & $-0.0066(8)$ & $0.0207(9)$ & $0.0000(9)$ \\
\hline$O(1)$ & $8 f$ & $0.5825(1)$ & $0.4055(1)$ & $0.9654(2)$ & $0.0376(8)$ & $0.0357(7)$ & $0.0293(7)$ & $0.0098(6)$ & $0.0018(6)$ & $-0.0038(6)$ \\
\hline$O(2)$ & $8 f$ & $0.5850(1)$ & $0.4899(1)$ & $1.1673(2)$ & $0.0414(8)$ & $0.0405(8)$ & $0.0283(7)$ & $0.0126(6)$ & $-0.0026(6)$ & $-0.0037(6)$ \\
\hline
\end{tabular}

Acknowledgments. We thank the Science Foundation for Young Teachers of Northeast Normal University (grant no. 20060304) and the Analysis and Testing Foundation of Northeast Normal University for support.

\section{References}

1. Zhang, W.-L.; Liu, Y.-Y.; Ma, J.-F.; Jiang, H.; Yang, J.: Syntheses and characterizations of nine coordination polymers of transition metals with carboxylate anions and bis(imidazole) ligands. Polyhedron 27 (2008) 3351-3358.

2. Yang, J.; Ma, J.-F.; Liu, Y.-Y.; Li, S.-L.; Zheng, G.-L.: Four Novel 3D Copper(II) Coordination Polymers with Different Topologies. Eur. J. Inorg. Chem. (2005) 2174-2180.

3. Qi, Y.; Luo, F.; Che, Y.; Zheng. J.: Hydrothermal Synthesis of MetalOrganic Frameworks Based on Aromatic Polycarboxylate and Flexible Bis(imidazole) Ligands. Cryst. Growth Des. 8 (2008) 606-611.
4. Zhang, L.-P.; Yang, J.; Ma, J.-F.; Jia, Z.-F.; Xie, Y.-P.; Wei, G.-H.: A series of 2D and 3D metal-organic frameworks based on different polycarboxylate anions and a flexible $2,2^{\prime}$-bis $(1 \mathrm{H}$-imidazolyl)ether ligand. CrystEngComm 10 (2008) 1410-1420.

5. Sheldrick, G. M.: SHFI XS-97. Program for the Solution of Crystal Structures. University of Göttingen, Germany 1997.

6. Sheldrick, G. M.: SHELXL-97. Program for the Refinement of Crystal Structures. University of Göttingen, Germany 1997. 